\title{
The INTERNATIONAL MISSION study: minimally invasive surgery in ovarian neoplasms after neoadjuvant chemotherapy
}

A Fagotti, ${ }^{1,2}$ S Gueli Alletti, ${ }^{1}$ G Corrado, ${ }^{3}$ E Cola, ${ }^{2}$ E Vizza, ${ }^{3}$ M Vieira,,${ }^{4}$ C E Andrade,${ }^{4}$ A Tsunoda,${ }^{4}$ G Favero, ${ }^{5}$ I Zapardiel, ${ }^{6}$ T Pasciuto, ${ }^{7}$ G Scambia ${ }^{1,2}$

\begin{abstract}
- There is an online supplementary infographic to go with this paper. To view it, please visit the journal website at https://ijgc.bmj.com/
\end{abstract}

For numbered affiliations see end of article.

Correspondence to S Gueli Alletti, Fondazione Policlinico Universitario A. Gemelli, Universita' Cattolica del Sacro Cuore, Rome, Italy; salvatore.guelialletti@ policlinicogemelli.it

Received 21 July 2018 Revised 23 September 2018 Accepted 25 September 2018

\section{SLinked}

- http://dx.doi.org/10.1136/ ijgc-2018-000001

Check for updates

(C) IGCS and ESGO 2019. № commercial re-use. See rights and permissions. Published by BMJ.

To cite: Fagotti A, Gueli Alletti S, Corrado G, et al. Int J Gynecol Cancer 2019;29:5-9.

\section{HIGHLIGHTS}

- Minimally invasive surgery was associated with a $96 \%$ rate of R0 resection at interval surgery.

- The rate of intraoperative complications when performing minimally invasive interval surgery was $4.7 \%$.

- Minimally invasive interval surgery may be considered in the setting of complete or partial response after neoadjuvant chemotherapy.

\section{ABSTRACT}

Objectives The aim of this retrospective multicenter study was to investigate the extent, feasibility, and outcomes of minimally invasive surgery at the time of interval debulking surgery in different gynecological cancer centers.

Methods/Materials In December 2016, 20

gynecological cancer centers were contacted by e-mail, to participate in the INTERNATIONAL MISSION study. Seven centers confirmed and five were included, with a total of 127 patients diagnosed with advanced epithelial ovarian cancer after neoadjuvant chemotherapy and minimally invasive interval surgery. Only women with a minimum follow-up time of 6 months from interval surgery or any cancer-related event before 6 months were included in the survival analysis. Baseline characteristics, chemotherapy, and operative data were evaluated. Survival analysis was evaluated using the Kaplan-Meier method.

Results All patients had optimal cytoreduction at the time of interval surgery: among them, $122(96.1 \%)$ patients had no residual tumor. Median operative time was 225 min (range $60-600$ ) and median estimated blood loss was $100 \mathrm{~mL}$ (range $70-1320$ ). Median time to discharge was 2 days (1-33) and estimated median time to start chemotherapy was 20 days (range $15-60)$. Six $(4.7 \%)$ patients experienced intraoperative complications, with one patient experiencing two serious complications (bowel and bladder injury at the same time). There were six (4.7\%) patients with postoperative short-term complications: among them, three patients had severe complications. The conversion rate to laparotomy was $3.9 \%$. Median follow-up time was 37 months (range 7 $86): 74$ of 127 patients recurred (58.3\%) and $31(24.4 \%)$ patients died from disease. Median progression-free survival was 23 months and survival at 5 years was $52 \%$ (95\% Cl: 35 to 67).

Conclusions Minimally invasive surgery may be considered for the management of patients with advanced ovarian cancer who have undergone neoadjuvant chemotherapy, when surgery is limited to low-complexity standard cytoreductive procedures.

\section{INTRODUCTION}

Ovarian carcinoma is the second most common gynecologic malignancy and the most common cause of death among women with gynecologic cancers. ${ }^{1}$ Women with advanced epithelial ovarian cancer have an expected long-term survival of approximately $20 \%{ }^{2}$ Standard treatment in advanced ovarian cancer is primary cytoreductive surgery followed by platinum-taxane chemotherapy, ${ }^{3}$ but over the past few decades, the use of interval surgery after neoadjuvant chemotherapy in patients with unresectable disease (International Federation of Gynecology and Obstetrics stage IIIC/ IV) or in patients with poor physical conditions, has been proposed to increase the rate of optimal debulking and reduce the number of complications, without affecting prognosis. ${ }^{4-7}$

Complete cytoreduction without macroscopic residual tumor, both as primary or interval surgery after neoadjuvant therapy is the aim of surgical management in patients with advanced ovarian cancer. In the past 5 years, technological advances, together with promising published results, has pushed the scientific community to make efforts to identify further innovative and personalized surgical treatments. ${ }^{89}$ However, a critical appraisal is needed regarding the surgical approach after neoadjuvant therapy. In fact, a variety of procedures are currently proposed at this time, ranging from standard cytoreductive procedures to the removal of all previously infiltrated tissues, including lymph nodes. ${ }^{10}$ Moreover, approaches may differ, with preliminary results showing minimally invasive surgery to be as effective as laparotomy at the time of interval surgery in patients with adequate response to chemotherapy. ${ }^{1112}$

In particular, the use of 'interval' minimally invasive surgery in the specific setting of advanced ovarian cancer patients after neoadjuvant therapy is still very sporadic and has not been completely examined. 


\section{Original Article}

Thus, the aim of this retrospective multicenter study was to investigate the extent, feasibility, and outcome of minimally invasive surgery at the time of interval surgery in different gynecological cancer centers.

\section{METHODS}

In December 2016, 20 gynecological cancer centers were asked to participate in a retrospective multicenter observational study, named the INTERNATIONAL MISSION study. The centers were selected based on their experience in minimally invasive surgery, ovarian cancer treatment, and data management as assessed by their scientific publications production. The Institutional Review Board of each participating institution approved the study, and all patients signed a written informed consent to collect their data prospectively and to analyze retrospectively.

Patients with advanced epithelial ovarian cancer treated with a minimally invasive surgery after neoadjuvant chemotherapy, either robotically or laparoscopically, were eligible for the study. Inclusion criteria were histologically proven advanced epithelial ovarian cancer (International Federation of Gynecology and Obstetrics stage III-IV); unresectable disease at primary surgery confirmed either by laparoscopy, laparotomy, or combined CT scan evaluation and cytology; neoadjuvant chemotherapy (any number of cycles); and interval surgery within 45 days after completion of chemotherapy. No specific exclusion criteria were adopted and each center could freely choose the proper medical and surgical treatment for each patient. All patients not receiving standard intraperitoneal cytoreduction and with follow-up time $<6$ months from interval surgery were excluded from the study. Standard cytoreduction was defined as all surgical procedures (hysterectomy, salpingo-oophorectomy, omentectomy, peritoneal biopsies) that are routinely performed at the time of interval surgery independent of the presence of tumor infiltration. Cancer centers with $<7$ patients were also excluded. Medical records were reviewed for baseline characteristics including age, International Federation of Gynecology and Obstetrics stage, body mass index, American Society of Anesthesiologists score, pathologic characteristic and chemotherapy details. Clinical response was assessed according to Response Evaluation Criteria in Solid Tumors. ${ }^{13}$ Surgical complications were graded according to the Memorial Sloan Kettering Cancer Center grading system, ${ }^{14}$ which categorizes the complications into five grades ranked $0-1$ : grade 1 (absent/minor complications not requiring therapy); grade 2 (complications requiring medications only without invasive procedures); grade 3 (complications leading to lasting disability or organ resection); grade 4 (life-threatening complications requiring intensive care unit stay); and grade 5 (death due to complications). Follow-up procedures were performed in accordance with National Comprehensive Cancer Network Clinical Practice Guidelines in Oncology. ${ }^{3}$ In the case of increased CA-125 serum levels and/ or clinical suspicion of recurrence, a CT scan and/or fluorodeoxyglucose-positron emission tomography scan were requested to confirm recurrence.

\section{Survival analysis}

Progression-free survival was defined as the time elapsed from initial diagnosis to relapse or last follow-up visit. Overall survival was calculated from the time of initial diagnosis to the date of death

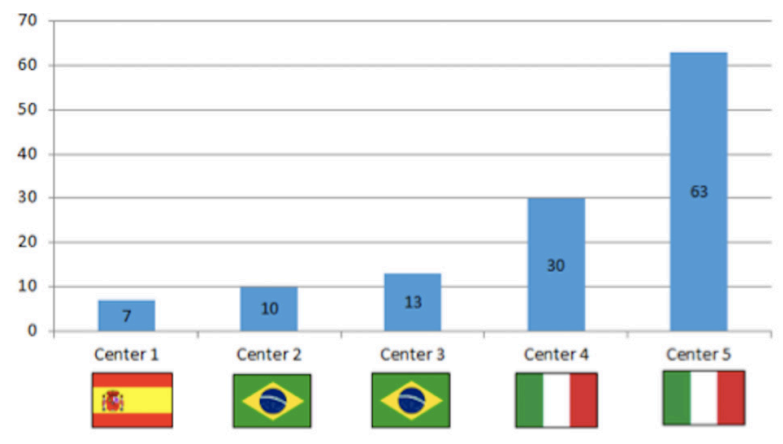

\begin{tabular}{|l|l|r|}
\hline Center 1 & La Paz University Hospital. & 7 \\
\hline Center 2 & Madrid, Spain & 10 \\
\hline & ICESP. São Paulo, Brazil & \\
Center 3 & $\begin{array}{l}\text { Barretos Cancer Hospital. São } \\
\text { Paulo, Brazil }\end{array}$ \\
\hline Center 4 & Regina Elena. Rome, Italy & 30 \\
\hline & Policlinico A. Gemelli. Rome, \\
Center 5 & Italy & 63 \\
\hline Total & & $\mathbf{1 2 3}$ \\
\hline
\end{tabular}

Figure 1 Number of patients enrolled in each center.

or last follow-up visit. Median follow-up was calculated according to the inverted Kaplan-Meier technique. ${ }^{15}$ Overall survival and PFS curves were estimated by the Kaplan- Meier product limit method. ${ }^{16}$ Only women with a minimum follow-up of 6 months from interval surgery or any cancer-related event before 6 months were included in the survival analysis. All statistical calculations were performed using the Stata software version 13.0 (Stata Corp, College Station, TX).

\section{RESULTS}

Of the 20 gynecological cancer centers asked to participate in the INTERNATIONAL MISSION study, seven centers agreed to participate and five were included, with an overall 127 consecutive patients (Figure 1). Patients were treated between July 2009 and July 2017: 94 patients (74\%) were deemed to have unresectable tumor volume at primary surgery by laparoscopy, 14 patients $(11 \%)$ were considered unresectable by laparotomy, and 19 patients (15\%) were deemed unresectable by combined evaluation with CT scan and cytology. Patient characteristics are included in Table 1. Median age was 60 years, obesity was found in $13.8 \%$ (median body mass index $24.7 \mathrm{~kg} / \mathrm{m}^{2}$, range 15.6-64). The histological type of ovarian cancer was high-grade serous in $90.6 \%$ of patients and most had stage IIIC disease (83.5\%), with ASA score of 2 (83.5\%).

All patients received neoadjuvant platinum-based chemotherapy with a median number of four cycles (range 3-8). An overall response rate of $96.8 \%$ was reported (38 patients [29.9\%] with a clinical complete response and 85 patients [66.9\%] with a clinical partial response) according to Response Evaluation Criteria in Solid Tumors. ${ }^{13}$ At final pathological diagnosis, complete response was observed in 27 patients and partial microscopic response in 19 additional patients (Table 2). Ninety-five of $127(72.5 \%)$ patients received adjuvant chemotherapy after interval surgery. All patients were optimally resected (residual tumor $<1 \mathrm{~cm}$ ) at the time of interval surgery: ${ }^{17}$ among them, $122(96.1 \%)$ patients had no residual tumor. Concomitant surgical procedures performed are 
Table 1 Patient characteristics.

\begin{tabular}{|c|c|}
\hline \multicolumn{2}{|c|}{ Patient's characteristics } \\
\hline Variable & No. $(\%)$ * \\
\hline All cases & 127 \\
\hline Median age (range) & $60(33-84)$ \\
\hline Median BMI (range)† & $24.7(15.6-64)$ \\
\hline From 17 to 24 & $63(51.2)$ \\
\hline From 25 to 27 & $31(25.2)$ \\
\hline From 28 to 30 & $12(9.8)$ \\
\hline Over 30 & $17(13.8)$ \\
\hline Previous laparotomies & $39(30.7)$ \\
\hline \multicolumn{2}{|l|}{ ASA score } \\
\hline 1 & 13 (10.2) \\
\hline II & $106(83.5)$ \\
\hline III & $8(6.3)$ \\
\hline \multicolumn{2}{|l|}{ Histotype (WHO) } \\
\hline High grade serous & $115(90.6)$ \\
\hline Clear cell & $5(3.9)$ \\
\hline Edometrioid & $6(4.7)$ \\
\hline Mucinous & $1(0.8)$ \\
\hline \multicolumn{2}{|l|}{ Grading } \\
\hline 1 & $0(0)$ \\
\hline 2 & $7(5.5)$ \\
\hline 3 & $120(94.5)$ \\
\hline \multicolumn{2}{|l|}{ Stage at diagnosis } \\
\hline III C & $106(83.5)$ \\
\hline IV & $21(16.5)$ \\
\hline
\end{tabular}

${ }^{*}$ Results are presented as $\mathrm{N}(\%)$ except where indicated.

${ }^{\dagger}$ Available in 123 cases.

BMI, body mass index.

described in Table 3. Median operative time was $225 \mathrm{~min}$ (range 60-600) and median estimated blood loss was $100 \mathrm{~mL}$ (range $70-1320$ ). Median time to discharge was 2 days (range 1-33) and estimated median time to start chemotherapy was 20 days (range $15-60)$.

Among the 127 patients included, six (4.8\%) had intraoperative complications, with one patient having two serious complications (bowel and bladder injury at the same time). There were five (3.9\%) postoperative short-term complications, however only three had grade 3 according to the Memorial Sloan Kettering Cancer Center grading system, consisting of two bowel fistulas and one pleural effusion. The conversion rate to laparotomy was $3.9 \%$ (five of $127)$ and reasons for conversion were surgical adhesions in three patients, one bowel resection, and one iliac artery injury (Table 4).

\section{Survival analysis}

Survival analysis was performed for all patients. Median follow-up was 37 months (range 7-86): 74 patients have recurred (58.3\%) and $31(24.4 \%)$ have died from the disease. The pattern of recurrence was mainly intraperitoneal (56 of $74,75.7 \%$ ), either exclusive or mixed with other sites (parenchymal and/or lymph nodal). The
Table 2 Chemotherapy details

\begin{tabular}{lc} 
Chemotherapy details & No. (\%) * \\
\hline Variable & 127 \\
\hline All cases & $4(3-8)$ \\
Median no. of cyles of NACT (range) & \\
Type of chemotherapy & \\
Carboplatin - paclitaxol (either weekly & $92(72.4)$ \\
or monthly) & $27(21.3)$ \\
Carboplatin - paclitaxol - bevacizumab & $8(6.3)$ \\
Other (platinum-based) & $32(2-2122$ \\
Median Ca-125 serum level (range) at & \\
IDS, Ul/ml & \\
RECIST response & $38(29.9)$ \\
Complete & $85(66.9)$ \\
Partial & $4(3.1)$ \\
Stable & \\
Pathological response & $27(21.3)$ \\
Complete & $100(78.7)$ \\
Partial & $19(24.7)$ \\
Partial micro† &
\end{tabular}

IDS, interval debulking surgery. RECIST, Response Evaluation Criteria in Solid Tumors.

${ }^{*}$ Results are presented as N (\%) except where indicated. †19 of 77 cases.

Table 3 Surgical data

\begin{tabular}{lc}
\hline Surgical data & No. (\%)* \\
\hline Variable & 127 \\
\hline All cases & \\
Type of surgery: & $122(96.1)$ \\
Hysteretomy +/-BSO† & $111(87.4)$ \\
Omentectomy & $50(39.4)$ \\
Regional peritonectomy & $38(29.9)$ \\
Pelvic/aortic lymphadenectomy & $8(6.3)$ \\
Appendectomy & $6(4.7)$ \\
Diaphragmatic stripping & $3(2.4)$ \\
Bowel resection & \\
Redidual tumor: & $122(96.1)$ \\
0 & $5(3.9)$ \\
$<1$ & $225(60-600)$ \\
Median OT (range) & $2(1-33)$ \\
Median discharge time, d (range) & $2(1.6)$ \\
Intra-operative blood transfusion & $100(70-1320$ \\
Median EBL, ml (range) & $20(15-60)$ \\
Estimated median TTC, d (range) &
\end{tabular}

${ }^{*}$ Results are presented as $\mathrm{N}(\%)$ except where indicated. OT, operation time. EBL, estimated blood loss. TTC, time to start chemotherapy.

†For five patients previous hysterectomy was performed. †lf adjuvant therapy was started (95/127 cases). 


\section{Original Article}

Table 4 Complications data

\begin{tabular}{lc}
\hline Complications data & No. (\%) * \\
\hline Variable & 127 \\
\hline All cases & $7(5.5)$ \\
Intraoperative complications † & \\
Type of Intraoperative complication & $3(42.8)$ \\
$\quad$ Bowel injury & $3(42.8)$ \\
Bladder injury & $1(14.4)$ \\
$\quad$ Vascular injury & $6(4.7)$ \\
Early PO complications MSKCC $\ddagger$ & $3(2.4)$ \\
$\quad$ Severe PO complication (G3-G5)§ & $5(3.9)$ \\
Conversion to LPT & \\
Reasons for conversion & $3(60.0)$ \\
$\quad$ Surgical adhesions & $1(20.0)$ \\
Bowel resection & $1(20.0)$ \\
$\quad$ lliac artery injury &
\end{tabular}

${ }^{*}$ Results are presented as $\mathrm{N}(\%)$.

†In six patients (one patient had two complications (bladder and bowel perforation)).

$\ddagger$ According to Memorial Sloan Kettering Cancer Center (MSKCC) grading system. ${ }^{12}$

§Three bowel perforations.

LPT, laparotomy; PO, post-operative.

median progression-free survival was 23 months and the survival at 5 years was $52.6 \%(95 \% \mathrm{Cl}$ : 35.2 to 67.3$)$ (Figure $2 \mathrm{~A}, \mathrm{~B})$.

\section{DISCUSSION}

Despite the increasing use of neoadjuvant chemotherapy and interval surgery in advanced epithelial ovarian cancer, recent literature contains only a few studies on the minimally invasive approaches, with limitations such as a small series of cases. ${ }^{11}{ }^{12} 18-20$ An analysis of the US National Cancer Database has

A

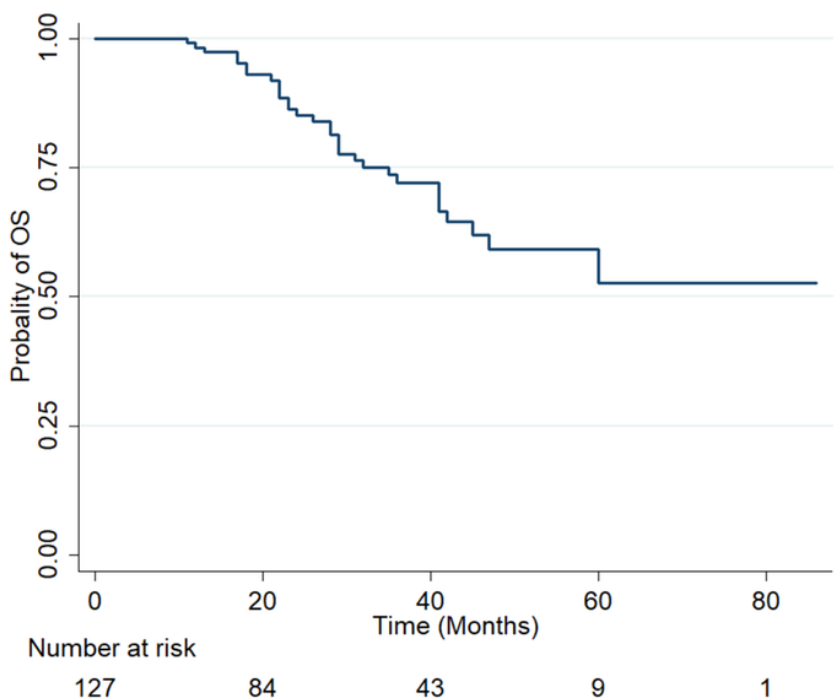

recently shown that patients with advanced ovarian cancer selected for laparoscopic interval surgery after neoadjuvant chemotherapy have similar perioperative outcomes and survival rates to women who undergo interval surgery by laparotomy. ${ }^{21}$ Conversely, the present study shows that minimally invasive surgery approaches are currently underused to perform interval surgery, even in some major centers with a focus on ovarian cancer treatment by laparoscopy or robotics.

Nevertheless, the current study demonstrated that minimally invasive interval surgery is a reasonable approach for patients, with advanced ovarian cancer after neoadjuvant chemotherapy who have had either complete or partial clinical response. The advantages of minimally invasive surgery compared with the traditional laparotomic technique ${ }^{22}$ are confirmed. Overall, the conversion rate of $3.9 \%$ is low, although higher than previously reported. ${ }^{11}$ This difference is probably the result of the broader inclusion criteria adopted, with no limitations in terms of body mass index, ASA score, or the number of chemotherapy cycles. The presence of extensive adhesions and gross residual disease after neoadjuvant chemotherapy, which are related to the high tumor burden at diagnosis, may also explain both conversion and complication rates in this series. Nevertheless, the safety of minimally invasive interval surgery is presented herein, with only six patients having an intraoperative complication (4.7\%) and three (2.4\%) patients experiencing severe early postoperative complications.

Another main goal of this study was to ensure that there was no adverse impact on patient survival. Critics may argue that a thorough peritoneal evaluation is not possible with minimally invasive surgery, resulting in undetected disease and worsened oncologic outcomes. Although eligibility to undergo interval surgery is related to response to neoadjuvant therapy, with an evident selection bias, the median progression-free and overall survival reported in this series seems reassuring regarding the oncologic safety of this approach (Figure 2).

We acknowledge that the short-term accrual and the heterogeneity of the patients, as well as their treatments in term of surgery and chemotherapy may represent potential limitations to this study.

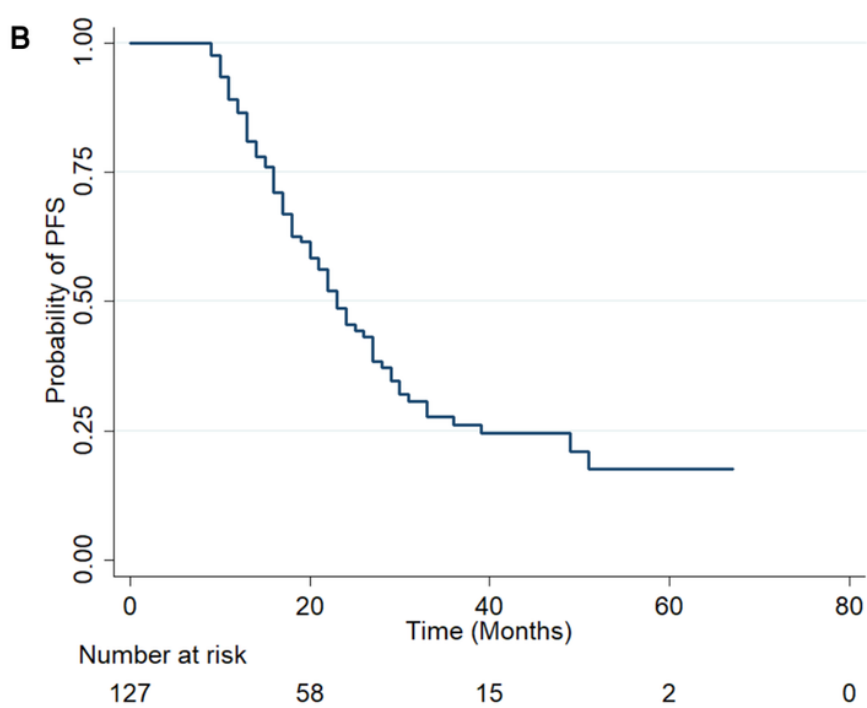

Figure 2 Progression-free $(A)$ and overall $(B)$ survival of patients treated with MIS after NACT, following a minimum follow-up time of 6 months after debulking or any cancer-related event before 6 months. 
Based on the current study, we suggest starting surgery with diagnostic laparoscopy and, if feasible, safely continuing by either laparoscopy or robotics to decrease the impact of aggressive surgery on high-morbidity patients, and thus reduce time to chemotherapy. ${ }^{12}$ This approach has also been found to be cost effective and can be used as a diagnostic tool before primary cytoreductive surgery in ovarian cancer. ${ }^{23}$ With this method, patients can be identified with insufficient response to NACT despite clinical evaluation, which can therefore lead to additional cycles of chemotherapy or to secondline therapy. ${ }^{24}$

Interval debulking surgery can integrate standard treatment, assess pathological response, and provides an opportunity to remove chemotherapy-resistant clones while improving quality of life in some cases. ${ }^{25}$ Recently published data have shown an acceptable outcome without interval surgery, suggesting a marginal role in women with good response to chemotherapy. ${ }^{26}$ In this complex scenario, a minimally invasive approach may be considered in the management of patients with advanced ovarian cancer who have undergone neoadjuvant chemotherapy, when surgery is limited to low-complexity cytoreductive procedures. Minimally invasive surgery allows surgeons to reserve the use of laparotomy for higher complexity procedures that may, in turn, be associated with a higher risk of complications.

\section{Author affiliations \\ ${ }^{1}$ Division of Gynecologic Oncology, Fondazione Policlinico Universitario A. Gemelli - IRCCS, Rome, Italy \\ 'Universita' Cattolica del Sacro Cuore, Rome, Italy \\ ${ }^{3}$ Department of Experimental Clinical Oncology, Gynecologic Oncology Unit, Regina Elena National Cancer Institute, Rome, Italy \\ ${ }^{4}$ Department of Gynecologic Oncology, Hospital de Cancer de Barretos, São Paulo, Brazil \\ ${ }^{5}$ Department of Gynecology, Instituto do Câncer do Estado de São Paulo-ICESP, Faculdade de Medicina da Universidade de São Paulo, São Paulo, Brazil ${ }^{6}$ Gynecologic Oncology Unit, La Paz University Hospital - IdiPAZ, Madrid, Spain ${ }^{7}$ Statistics Technology Archiving Research (STAR) Center, Fondazione Policlinico Universitario A. Gemelli - IRCCS, Rome, Italy}

Acknowledgements The authors wish to thank the following contributors: Dr. Minig L (Department of Gynecology, Instituto Valenciano de Oncología (IVO), Valencia, Spain) and Dr. Kim TJ (Department of Obstetrics and Gynecology, Samsung Medical Center, Sungkyunkwan University School of Medicine, Seoul, Korea).

Competing interests None declared.

Patient consent Not required.

Provenance and peer review Not commissioned; externally peer reviewed.

\section{REFERENCES}

1. Siegel RL, Miller KD, Jemal A. Cancer Statistics, 2017. CA Cancer J Clin 2017;67:7-30.

2. Hennessy BT, Coleman RL, Markman M. Ovarian cancer. Lancet 2009;374:1371-82.

3. NCCN Clinical Practice Guidelines in Oncology, 2017. Ovarian Cancer including Fallopian Tube Cancer and Primary Peritoneal Cancer. Version 3. Available from: https://www.nccn.org/ professionals/physician_gls/pdf/ovarian.pdf

4. Vergote I, Tropé CG, Amant F, et al. Neoadjuvant chemotherapy or primary surgery in stage IIIC or IV ovarian cancer. N Engl J Med 2010;363:943-53.

5. Kehoe S, Hook J, Nankivell M, et al. Primary chemotherapy versus primary surgery for newly diagnosed advanced ovarian cancer
(CHORUS): an open-label, randomised, controlled, non-inferiority trial. Lancet 2015;386:249-57.

6. Onda T, Satoh T, Saito T, et al. Comparison of treatment invasiveness between upfront debulking surgery versus interval debulking surgery following neoadjuvant chemotherapy for stage III/ IV ovarian, tubal, and peritoneal cancers in a phase III randomised trial: Japan Clinical Oncology Group Study JCOG0602. Eur J Cancer 2016;64:22-31.

7. Fagotti A, Ferrandina G, Vizzielli G, et al. Phase III randomised clinical trial comparing primary surgery versus neoadjuvant chemotherapy in advanced epithelial ovarian cancer with high tumour load (SCORPION trial): final analysis of peri-operative outcome. Eur J Cancer 2016;59:22-33.

8. Fagotti A, Costantini B, Gallotta V, et al. Minimally invasive secondary cytoreduction plus HIPEC versus open surgery plus HIPEC in isolated relapse from ovarian cancer: a retrospective cohort study on perioperative outcomes. J Minim Invasive Gynecol 2015;22:428-32.

9. Cianci S, Vizzielli G, Fagotti A, et al. A novel HIPEC technique using hybrid $\mathrm{CO}_{2}$ recirculation system: intra-abdominal diffusion test in a porcine model. Updates Surg 2018;70:529-33.

10. Fagotti A, De laco P, Fanfani F, et al. Systematic pelvic and aortic lymphadenectomy in advanced ovarian cancer patients at the time of interval debulking surgery: a double-institution case-control study. Ann Surg Oncol 2012;19:3522-7.

11. Gueli Alletti S, Bottoni C, Fanfani F, et al. Minimally invasive interval debulking surgery in ovarian neoplasm (MISSION trial-NCT02324595): a feasibility study. Am J Obstet Gynecol 2016;214:503.e1-6.

12. Gueli Alletti S, Petrillo M, Vizzielli G, et al. Minimally invasive versus standard laparotomic interval debulking surgery in ovarian neoplasm: a single-institution retrospective case-control study. Gynecol Oncol 2016;143:516-20.

13. Eisenhauer EA, Therasse $\mathrm{P}$, Bogaerts $\mathrm{J}$, et al. New response evaluation criteria in solid tumours: revised RECIST guideline (version 1.1). Eur J Cancer 2009;45:228-47.

14. Strong VE, Selby LV, Sovel M, et al. Development and assessment of Memorial Sloan Kettering Cancer Center's Surgical Secondary Events grading system. Ann Surg Oncol 2015;22:1061-7.

15. Schemper M, Smith TL. A note on quantifying follow-up in studies of failure time. Control Clin Trials 1996;17:343-6.

16. Kaplan EL, Meier P. Nonparametric estimation from incomplete observations. J Am Stat Assoc 1958;53:457-81.

17. van der Burg ME, Vergote I, Gynecological Cancer Group of the EORTC. The role of interval debulking surgery in ovarian cancer. Curr Oncol Rep 2003;5:473-81.

18. Nezhat FR, Finger TN, Vetere $P$, et al. Comparison of perioperative outcomes and complication rates between conventional versus robotic-assisted laparoscopy in the evaluation and management of early, advanced, and recurrent stage ovarian, fallopian tube, and primary peritoneal cancer. Int J Gynecol Cancer 2014;24:600-7.

19. Corrado G, Mancini E, Cutillo G, et al. Laparoscopic debulking surgery in the management of advanced ovarian cancer after neoadjuvant chemotherapy. Int J Gynecol Cancer 2015;25:1253-7.

20. Favero G, Macerox N, Pfiffer T, et al. Oncologic concerns regarding laparoscopic cytoreductive surgery in patients with advanced ovarian cancer submitted to neoadjuvant chemotherapy. Oncology 2015;89:159-66.

21. Melamed A, Nitecki R, Boruta DM, et al. Laparoscopy compared with laparotomy for debulking ovarian cancer after neoadjuvant chemotherapy. Obstet Gynecol 2017:129:861-9.

22. Medeiros LR, Stein AT, Fachel J, et al. Laparoscopy versus laparotomy for benign ovarian tumor: a systematic review and metaanalysis. Int J Gynecol Cancer 2008;18:387-99.

23. van de Vrie R, van Meurs HS, Rutten MJ, et al. Cost-effectiveness of laparoscopy as diagnostic tool before primary cytoreductive surgery in ovarian cancer. Gynecol Oncol 2017;146:449-56.

24. Fagotti A, Fanfani F, Vizzielli G, et al. Should laparoscopy be included in the work-up of advanced ovarian cancer patients attempting interval debulking surgery? Gynecol Oncol 2010;116:72-7.

25. Gueli Alletti S, Vizzielli G, Lafuenti L, et al. Single-institution propensity-matched study to evaluate the psychological effect of minimally invasive interval debulking surgery versus standard laparotomic treatment: from body to mind and back. J Minim Invasive Gynecol 2018;25:816-22.

26. Marchetti C, Kristeleit R, McCormack M, et al. Outcome of patients with advanced ovarian cancer who do not undergo debulking surgery: a single institution retrospective review. Gynecol Oncol 2017;144:57-60. 